\title{
Nutrição e mudanças alimentares em meio a pandemia COVID-19
}

\author{
Nutrition and food changes in the middle of the pandemic COVID-19 \\ Nutrición y cambios alimentarios en médio de la pandemia COVID-19
}

\author{
Gabrielle da Cruz Leão \\ ORCID: https://orcid.org/0000-0002-2497-8391 \\ Centro Universitário FAMETRO, Brasil \\ E-mail: gabby18tuan@gmail.com \\ José Carlos de Sales Ferreira \\ ORCID: https://orcid.org/0000-0002-1867-8229 \\ Centro Universitário FAMETRO, Brasil \\ E-mail: jcarlos.sales@gmail.com
}

\begin{abstract}
Resumo
Introdução: A COVID-19 também conhecida como coronavirus, é o vírus que tem afligindo toda população mundial. Por isso, se faz necessário conhecer os impactos causados pelas mudanças alimentares que afetaram a nutrição e a segurança alimentar nesse cenário de pandemia. Desta forma, foi realizado um levantamento bibliográfico com literaturas pesquisadas no Google Acadêmico, sendo selecionados dez artigos dentro do tema proposto. Demonstrou que as restrições causadas pelo isolamento social atingiram os indivíduos psicológica e mentalmente, repercutindo diretamente nos hábitos alimentares, pois houve o aumento no consumo de alimentos ultraprocessados, diminuição de consumo de alimentos in natura, redução das atividades físicas, ganho de peso. Estes aspectos provocam a má nutrição, consequentemente a desnutrição e a obesidade. Desta forma, o sistema imunológico é atingindo, gerando desfechos agravantes em pacientes com COVID-19. Diante do exposto é necessário ter um acompanhamento do nutricionista, para obter orientações da boa conduta alimentar aliada com a pratica de exercícios físicos que podem ajudar no metabolismo a obter reações positivas ao tratamento dessa patologia.
\end{abstract}

Palavras-chave: COVID-19; Nutrição; Mudanças Alimentares; Isolamento Social.

\begin{abstract}
Introduction: COVID-19, also known as coronavirus, is the virus that has afflicted the entire world population. Therefore, it is necessary to know the impacts caused by dietary changes that have affected nutrition and food security in this pandemic scenario. In this way, a bibliographic survey was carried out with literature researched on Google Scholar, with ten articles selected within the proposed theme. It demonstrated that the restrictions caused by social isolation affected individuals psychologically and mentally, directly affecting eating habits, as there was an increase in the consumption of ultra-processed foods, a decrease in the consumption of fresh foods, a reduction in physical activities, weight gain. These aspects cause malnutrition, consequently malnutrition and obesity. Thus, the immune system is reaching, generating aggravating outcomes in patients with COVID-19. In view of the above, it is necessary to have a follow-up from the nutritionist, to obtain guidance on good eating behavior combined with the practice of physical exercises that can help metabolism to obtain positive reactions to the treatment of this pathology.
\end{abstract}

Keywords: COVID-19; Nutrition; Food Changes; Social isolation.

\section{Resumen}

Introducción: COVID-19, también conocido como coronavirus, es el virus que ha afectado a toda la población mundial. Por tanto, es necesario conocer los impactos provocados por los cambios dietéticos que han afectado la seguridad alimentaria y nutricional en este escenario pandémico. De esta manera, se realizó un relevamiento bibliográfico con literatura investigada en Google Scholar, con diez artículos seleccionados dentro de la temática propuesta. Demostró que las restricciones provocadas por el aislamiento social afectaron psicológica y mentalmente a las personas, afectando directamente los hábitos alimentarios, ya que hubo un aumento en el consumo de alimentos ultraprocesados, una disminución en el consumo de alimentos frescos, una reducción en las actividades físicas y aumento de peso. Estos aspectos provocan desnutrición, consecuentemente desnutrición y obesidad. Así, el sistema inmunológico está alcanzando, generando resultados agravantes en pacientes con COVID-19. En vista de lo anterior, es necesario contar con un seguimiento por parte del nutricionista, con el fin de obtener pautas de buena conducta alimentaria combinada con la práctica de ejercicios físicos que puedan ayudar al metabolismo a obtener reacciones positivas al tratamiento de esta patología.

Palabras clave: COVID-19; Nutrición; Cambios Alimentarios; Aislamiento Social. 


\section{Introdução}

Nos dias atuais sabe-se que saúde não está relacionada a apenas a ausência de doenças, mas também está envolvendo o bem-estar físico, mental e social da população. Um dos determinantes da saúde é a alimentação. Segundo o Guia Alimentar da população Brasileira, a alimentação adequada e saudável é um direito humano básico que envolve a garantia ao acesso permanente e regular, de forma socialmente justa, a uma prática alimentar adequada aos aspectos biológicos e sociais do indivíduo, nos revelando a importância de práticas alimentares apropriadas ao ser humano, mantendo a harmonia em relação à quantidade e qualidade, e que atendam aos princípios da variedade, equilíbrio, moderação e prazer (Brasil, 2014). Assim como a ausência da ingestão de alimentos causam efeitos negativos, como a desnutrição, a ingestão excessiva pode levar a condições como o sobrepeso e a obesidade. Ainda, podendo ocasionar outras doenças pela contaminação dos alimentos e o agravamento de outras doenças crônicas (Brasil, 2020).

A Síndrome Respiratória Aguda Grave - COVID-19, também conhecido como coronavírus, é causada pelo vírus SARS-CoV-2 (do inglês Severe Acute Respiratory Syndrome Coronavirus 2), que se diz ser de origem zoonótica. No final de dezembro de 2019, o surto da COVID-19 ocorreu na província de Hubei, na China, levando à rápida disseminação para muitos países do mundo (Abbas, 2020) como consequência a Organização Mundial da Saúde (OMS) a caracterizou como uma pandemia. Esta nova crise de saúde pública que ameaça a humanidade, causada pelo novo coronavírus ou COVID-19, está marcando a história trazendo várias mudanças no cenário mundial.

Devido a esta situação, os governos adotaram medidas de distanciamento físico, visto que o maior meio de transmissão da doença infecciosa é por meios diretos como: gotículas respiratórias de pessoas contaminadas e transmissão de pessoa para pessoa; e por contato indireto como: objetos contaminados e contágio aéreo (Butler, 2020). As pessoas de diferentes partes do mundo foram orientadas a tomar medidas preventivas como o isolamento social, limitando suas saídas a necessidades estritas, pois esta atitude tem sido a resposta mais eficaz a tal situação, defendida pelas autoridades sanitárias de todo o mundo e adotada pela imensa maioria dos governos mundiais na tentativa de reduzir o nível de letalidade exponencialmente (Bicalho, 2020).

Segundo a OMS (Organização Mundial da Saúde) não existe, até ao momento, evidência de qualquer tipo de contaminação através do consumo de alimentos cozidos ou crus. Alimentos não são vetores do vírus, denominado Corona, mas a falta dele pode comprometer a resistência de indivíduos com relação às doenças infecciosas, em geral. A alimentação saudável fornece todos os nutrientes necessários ao funcionamento satisfatório do nosso corpo, porém, a carência de essenciais nutrientes em detrimento do não consumo de determinados grupos alimentares poderá provocar carências nutricionais, por vezes irreversíveis, daí a importância do equilíbrio alimentar (Conte, 2020).

O presente estudo objetiva realizar um levantamento na literatura sobre os impactos causados devido às mudanças alimentares relacionadas ao COVID-19 e de como essas mudanças afetaram a nutrição e a segurança alimentar.

\section{Metodologia}

Este trabalho trata-se de um levantamento bibliográfico, consultados nas bases do Google Acadêmico no ano de 2020 e 2021. Como critérios de inclusão, foram consideradas as produções da literatura no referido período, disponíveis na íntegra online e selecionados os artigos na língua inglesa e portuguesa. Os critérios de exclusão foram artigos que não se adequaram ao tema proposto. A partir desse levantamento foram selecionados 10 artigos que incluíram estudos transversais, estudos de coorte e revisões bibliográficas que relacionavam o COVID-19 com a nutrição e as mudanças alimentares. Como respaldo para a interpretação e análise dos artigos selecionados foi utilizado a abordagem qualitativa com metodologia descrita por Pereira et al. (2018). 
O embasamento teórico foi realizado em sites de busca de artigos indexados no Google Acadêmico, com artigos e revisões científicas sobre a nutrição e as mudanças nos comportamentos alimentares, nesse período da pandemia COVID-19. Além disso, foram incluídos dados oficiais divulgados em sites de órgãos governamentais.

As produções da literatura que foram utilizadas para sustentar esta análise compreenderam os critérios mencionados entre os anos de 2020 a 2021 com as palavras-chave "COVID-19", "nutrição", com os seguintes subtópicos: "mudança alimentar", "micronutrientes", "imunidade", "segurança alimentar" disponíveis na íntegra online e selecionados nos idiomas inglês e português.

\section{Resultados e Discussão}

Através da análise feita dos 10 artigos selecionados, foi possível destacar alguns impactos causados pela pandemia COVID-19, referentes às mudanças alimentares e à nutrição.

Estudos demonstram que, as incertezas sobre como controlar a COVID-19 e sobre sua gravidade, além da imprevisibilidade acerca do tempo de duração dessa pandemia e dos seus desdobramentos, caracterizam-se como fatores de risco à saúde mental da população geral (Zandifar \& Badrfam, 2020). Este assunto também é enfatizado por Oliveira e colaboradores (2021), que diz que essa baixa previsibilidade e a incerteza dessa doença ameaçam não só a saúde física das pessoas, como também a saúde mental, especialmente em termos de emoções e cognição. Já Schmidt et al (2020) propõe que à saúde emocional, tende a ser negligenciada em um primeiro momento, pois em regra geral durante pandemias a saúde física das pessoas e o combate do agente patogênico são os focos primários dos gestores e profissionais da saúde.

Outros estudos apontam que estas respostas psicológicas e emocionais desencadeadas pela pandemia podem aumentar o risco de desenvolver uma alimentação disfuncional, prejudicando o comportamento pessoal (Li et al., 2020; Montemurro 2020; Wang et al., 2020; Oliveira et al, 2021).

Ao se referir nas consequências causadas pelas mudanças de hábitos alimentares, o estudo de Oliveira e colaboradores (2021), ainda destaca que essas mudanças de hábitos causadas por esse cenário, bem como suas consequências, estão sendo alvo de muitos estudos na atualidade que objetivam entender os efeitos causados, principalmente, pelo isolamento social para a população mundial. Além disso, para muitos indivíduos, essa pandemia está associada à alteração da rotina de trabalho, que pode resultar em situações emocionais como tédio, estresse e ansiedade, que afetam diretamente o consumo de alimentos como maior ingestão de energia, ricos em gorduras e açúcar e a chamada comfort food (Araújo et al., 2020). Este aumento do estresse, em situação de quarentena, já esteve associado às mudanças alimentares (Rolland et al., 2020), como a piora na qualidade da dieta (Schweren et al., 2020), o que significa que essas mudanças alimentares acarretam vários outros problemas relacionados à saúde (Martinez Steele et al., 2020).

Essas implicações causadas pela pandemia do COVID-19, por sua vez, estão ligadas as mudanças no estilo de vida que mais adiante podem trazer consequências nos aspectos psicológicos e emocionais, isso também é exposto em pesquisa realizada por Durães e colaboradores (2020), que revela que estes aspectos estão refletindo diretamente na dieta e na mudança negativa dos hábitos alimentares. Ressaltou que a produção, comercialização, oferta e consumo de alimentos foram afetados pelo isolamento social, além das mudanças no meio econômico, estes fatores atingiram de forma significante a população mais vulnerável em termos sociais, econômicos e sanitários, no que se refere à oferta suficiente de alimentos in natura (frutas, legumes, vegetais) e um aumento no consumo de alimentos ultraprocessados em virtude do preço e da facilidade de acesso (Ribeiro-Silva et al., 2020; Durães et al., 2020).

Ainda em se tratar de aspectos associados à pandemia que influenciaram a qualidade da alimentação, Demoliner e Daltoe (2020) ressaltou em sua pesquisa que entre esses aspectos estão o aumento do consumo de uma dieta rica em gordura 
saturada, carboidratos refinados, álcool, e baixos níveis de fibra, gordura insaturada, micronutrientes e antioxidantes que prejudicam significativamente a imunidade adaptativa enquanto aumenta a imunidade inata, levando a inflamação crônica e prejudicando gravemente a defesa do hospedeiro contra patógenos virais, o que pode representar um risco em dimensões maiores para a patologia grave da COVID-19 em grupos de risco (Zang et al, 2020, Demoliner \& Daltoe, 2020)

Ao se comparar os trabalhos de Durães et al. (2020) e Demoliner e Daltoe (2020) ambos enfatizam que uma dieta desequilibrada, com baixo consumo de alimentos in natura e minimamente processados, além do alto consumo de produtos industrializados podem trazer consequências sérias como obesidade e doenças crônicas como hipertensão, diabetes, doenças cardíacas, doenças pulmonares.

Esta situação foi observada por Lopes et al (2020) que mostrou os fatores de mudanças do comportamento alimentar e que colaboraram para modificações no perfil epidemiológico nutricional no Brasil, processo reconhecido como transição nutricional, no qual doenças relacionadas ao excesso de peso, como obesidade, diabetes e hipertensão, sobrepuseram-se às doenças relacionadas às carências nutricionais (Vasconcelos \& Batista Filho, 2011; Lopes 2020). Deste modo, existe uma maior preocupação em relação ao aumento do consumo de alimentos ultraprocessados, já que é uma das principais causas da atual pandemia de obesidade e de agravos não transmissíveis, pois esses produtos possuem mais calorias, apresentam maior quantidade de açúcar livre, sódio, gorduras totais e gorduras saturadas e menor teor de proteínas e fibras, quando comparados aos alimentos in natura ou minimamente processados (Monteiro et al., 2010; Louzada et al., 2015; Lopes, 2020).

Prosseguindo essa linha sobre alimentos ultraprocessados, uma pesquisa desenvolvida na NutriNet BrasiL, de Martínez Steele et al (2020) demonstrou em seu resultado uma certa preocupação em relação a tendência do aumento da ingestão desses tipos de alimentos, principalmente, observadas nas regiões Nordeste e Norte e entre pessoas com menor escolaridade, nesse período de pandemia do COVID-19. Porém, isso instiga a realizar novas investigações, pois se comprovados tais fatos, isto impele certos cuidados, uma vez que a ingestão desses alimentos ascende o risco de patologias como obesidade, hipertensão e diabetes (Claro et al., 2015; Elizabeth et al., 2020; Martínez Steele et al., Oliveira et al., 2021), elevando assim a probabilidade de desenvolvimento de formas graves da COVID-19 (Cai et al., 2020, Costa et al., 2020).

Outra pesquisa desenvolvida no Brasil, no Centro Universitário do Sul, por Martinotto e colaboradores (2020) analisaram os hábitos alimentares dos funcionários desse Centro, constatou-se que dentre os indivíduos avaliados 34,7\% pioraram a qualidade da alimentação, 29\% aumentaram a ingestão de frutas e/ou vegetais e 20,8\% passaram a consumir alimentos industrializados. Esta pesquisa foi comparada com outras feitas neste período como o de Pellegrini et al. (2020), onde $27,3 \%$ dos participantes relataram comer mais frutas e/ou vegetais do que antes do período da pandemia, 17,3\% consumiram mais alimentos enlatados/congelados e 50\% aumentaram o consumo de doces no referido momento (Pellegrini et al., 2020). Demonstrando assim, importantes mudanças nos hábitos alimentares, como piora na qualidade da alimentação, aumento no consumo de alimentos industrializados, no entanto também mostrou o aumento do consumo de frutas e/ou vegetais (Martinotto et al., 2020).

O estudo de Araújo e colaboradores (2020) reforça que a nutrição saudável se torna uma prioridade no momento atual tanto para o aspecto da população psicológica e emocionalmente afetados, principalmente, sob estresse devido à quarentena, assim como também, para as pessoas que foram afetadas pela COVID-19 com sintomatologia leve e/ou assintomática. Ainda ressalta que a partir da alimentação equilibrada em nutrientes, aliada a adequada hidratação e horas de sono, auxiliam no fortalecimento da imunidade e dando ao organismo maiores chances de combater e se proteger contra esse vírus. Mas, ainda não é possível comprovar que exista um alimento específico ou uma conduta nutricional que combata a contaminação, porém é de suma importância o auto cuidado e a manutenção do estado de saúde saudável (Lima, 2020; Araújo et al, 2020). Por isso, a segurança alimentar e nutricional é também citada por Durães et al. (2020), pois a má nutrição pode afetar diretamente na 
redução das defesas do organismo, tornando a população mais suscetível ao adoecimento, principalmente, os idosos e portadores de patologias crônicas (Brasiel, 2020).

Uma alimentação saudável pode auxiliar, não só por fornecer os nutrientes e compostos bioativos necessários para a integridade da barreira imunológica, mas também por garantir a manutenção do peso saudável (Misumi et al, 2019). Esse aspecto em particular, tem sido relatado na literatura como relevante, pois a má nutrição pode ocasionar tanto a desnutrição como a obesidade, estas por sua vez, estão associadas a piores desfechos em pacientes acometidos pela COVID-19, com o maior risco de hospitalização, maior tempo de internação e mortalidade (Araújo et al, 2020).

Outros trabalhos como o de Mendes e colaboradores (2020), evidencia que nesta pandemia, e em futuras situações, podem-se enfrentar complicações por desnutrição com uma perspectiva antagônica de ser vítima da desnutrição por carência, malnutrição e em simultâneas vítimas da desnutrição por excesso de nutrição com situações de pré-obesidade/obesidade. E com base em literatura disponível revela que propostas são recomendadas de maneira objetiva e sistematizadas oferecendo o suporte nutricional para doentes com COVID-19, como as da European Society for Clinical Nutrition and Metabolism (Espen), que visam à prevenção e tratamento da malnutrição associada à COVID-19. Esta associação ao coronavírus revela-se como um fator de risco independente no aumento da mortalidade (Barazzoni et al., 2020; Mendes et al., 2020). Salienta ainda, que a intervenção e a terapia nutricionais devem ser consideradas parte integrante da abordagem dos doentes vítimas desse vírus, em especial, nos doentes idosos, frágeis e com comorbidades (Mendes et al., 2020).

Outro fator preocupante nesse sentido está relacionado à situação nutricional de crianças e adolescentes, pois estudos como o de Sousa e colaboradores (2020) e Costa e colaboradores (2020) estão direcionados a repercussões decorrentes da COVID-19 na epidemia da obesidade de crianças e adolescentes. Sousa e colaboradores (2020) destaca que na situação econômica atual, o sustento de muitas famílias ficou comprometido e a disponibilidade de alimentos nutritivos ficou ainda mais escasso o que impactou diretamente na alimentação. Trazendo como consequência o maior consumo de alimentos processados e enlatados que são mais fáceis de adquirir e armazenar e possuem maior prazo de validade. Esses tipos de alimentos têm baixo valor nutricional e alto valor calórico, contribuindo para o surgimento ou agravamento de sobrepeso e obesidade (Sociedade Brasileira de Pediatria 2020). Destacou outro impacto que refere-se à redução das atividades físicas das crianças e adolescentes pois os mesmos não dispõem de local adequado para brincadeiras ou realizações de atividades que permitam a diminuição ou o término do sedentarismo (Cabrera et al., 2020; Sousa et al., 2020).

Já Costa e colaboradores (2020) atestam que as comorbidades pré-resistentes parecem ser fator importante na gravidade dos casos e, dentre elas, a obesidade estar emergindo como possível fator de risco para ocorrência de casos de COVID-19, principalmente em crianças mais velhas (Sinha et al., 2020; Shenkerdemia et al., 2020). Embora a prioridade à frente da pandemia da doença pelo COVID-19 seja considerar o seu impacto imediato, é necessário chamar atenção para o efeito dessa pandemia em longo prazo, em especial na saúde das crianças e adolescentes. Com as medidas que visam o distanciamento social, feitas pelas autoridades e a permanência em casa, as oportunidades de atividades físicas entre as crianças e adolescentes diminuiu consideravelmente, ou seja, elas se exercitam menos (Ribeiro et al., 2020). Além disso, o consumo de alimentos industrializados vem aumentando ao longo desse período, assemelhando-se ao período de férias escolares (Ribeiro et al., 2020; Rundle et al., 2020).

Esses encadeamentos demonstram que se devem ficar mais atentos a essa situação tão prejudicial à saúde. Ao mencionar assuntos referentes ao aumento de comportamentos de risco a saúde, Malta e colaboradores (2020), apontam as reduções da atividade física e do consumo de alimentos saudáveis, além do alto consumo dos ultraprocessados. Relacionou esse estudo com outros desenvolvidos em outros países, confirmando a piora dos fatores de risco comportamentais. No que diz respeito à redução da prática de atividade física ressalta que isso pode provocar problemas cardiovasculares, entre outros (Monteiro et al., 2019; Peçanha et al., 2020). Já em relação ao aumento do consumo de alimentos ultraprocessados também 
constatou prejuízos a saúde como obesidade, hipertensão e doenças cardiovasculares (Elliston et al., 2017; Bhutani \& Cooper, 2020; Sallis et al., 2020).

Devido a todas essas mudanças, pesquisas como a de Dias e colaboradores (2020) e Ribeiro-Silva e colaboradores (2020), consideram que o estado nutricional, o estilo de vida, alimentação juntamente com a segurança alimentar, são de suma importância diante dessa pandemia. Dias e colaboradores (2020) dão ênfase na preservação do estado nutricional, além da prevenção ou tratamento da má nutrição, para reduzir complicações e resultados negativos em doentes com risco nutricional que possam contrair COVID-19 no futuro e/ou pacientes infectados (Zabetakis et al., 2020). Menciona que uma estratégia a ser adotada é controlar a atividades de mediadores inflamatórios por meio de fatores de risco modificáveis como a dieta, o exercício e escolhas saudáveis de estilo de vida. Destaca ainda que um adicional efeito da má nutrição sobre as infecções virais é o prolongamento do tempo de infecção e tempo de internação hospitalar, quando necessária. Além disso, reforça que as deficiências de micronutrientes afetam negativamente a função imune e podem diminuir a resistência às infecções, atingindo assim, o sistema imunológico (Calder et al., 2020). Desta forma, faz-se necessário a ingestão de nutrientes por meio do consumo de uma dieta equilibrada e diversificada, podendo exigir uma ingestão acima da RDA (Recommended Dietary Allowance - Ingestão Dietética Recomendada) para alguns micronutrientes.

Enquanto, Ribeiro-Silva e colaboradores (2020) consideram, em termos gerais, duas dimensões que foram afetadas pela pandemia COVID-19, são elas: a alimentar e a nutricional, sendo que a primeira se refere ao processo de disponibilidade (produção, comercialização e acesso ao alimento); a segunda estende-se a escolha, ao preparo e ao consumo alimentar e sua relação com a saúde e com a utilização biológica do alimento. Ambas visam garantir alimentos saudáveis, que respeitem a cultura alimentar da população e que tenham sido produzidas de forma sustentável.

Nos achados de Ribeiro-Silva e colaboradores (2020) há pontos, em comum, com outras pesquisas já aqui mencionadas, alerta sobre práticas para reorientar as pessoas em busca da boa nutrição, principalmente, no que tange a respeito ao isolamento social que trouxe consigo importantes mudanças socioculturais, redução da atividade física e alterações os hábitos alimentares, determinantes essenciais ao estado nutricional. Enfatizou que os hábitos alimentares são afetados por condições de angústias e distúrbios emocionais, que em níveis elevados são associados à má qualidade da dieta (Naja \& Hamadeh, 2020). Expôs que é preciso criar alternativas que respeitem o Guia Alimentar dos Brasileiros, 2014, recomendado pelo Ministério da Saúde, e que protejam as pessoas da má nutrição (Brasil, 2014), além de ações alinhadas ao enfrentamento dessa situação sem desconsiderar a insegurança alimentar nas diversas dimensões.

\section{Conclusão}

A pandemia da COVID-19 trouxe consigo várias mudanças e com base nos dados expostos nas literaturas estudadas constatou-se que essas modificações no comportamento e estilo de vida das pessoas sofreram inúmeros impactos, aumentando o risco dessa doença. Essas mudanças estão relacionadas às restrições provocadas pela pandemia, como o isolamento social, ocasionando problemas psicológicos e emocionais, que refletiram nos maus hábitos alimentares, na redução de atividade física e o agravamento de doenças crônicas.

Os pontos negativos mais destacados se referem ao alto índice no consumo de alimentos industrializados e ultraprocessados, estes por sua vez, são alimentos de baixo valor nutricional e alto valor calórico, o que ocasiona a má nutrição, consequentemente, a desnutrição e a obesidade, agravantes da COVID-19. Os estudos enfatizam que é essencial ter um acompanhamento nutricional, pois ter uma dieta saudável, equilibrada e diversificada, tem influência no sistema imunológico que pode ajudar no combate a esse vírus. Optar por um estilo de vida saudável aliada com exercícios físicos, 
alimentação equilibrada, dar preferência ao consumo de alimentos in natura, seguir orientações de boa nutrição, são algumas das recomendações mais destacadas nas literaturas pesquisadas.

Como a nutrição tornou-se um fator imprescindível na recuperação e tratamento de muitos pacientes, não isoladamente, mas como suporte que está diretamente ligado ao sistema imunológico. Sugere-se que estudos futuros sejam realizados visando aprofundar conhecimentos sobre o assunto, pois a pandemia afetou vários setores da sociedade, trazendo consigo vários agravantes para saúde da população. Esses estudos podem auxiliar os demais trabalhos que estão em andamento, aperfeiçoando-os, e no desenvolvimento de outros, agregando conhecimentos para as equipes multiprofissionais da saúde.

\section{Referências}

Abbas, A. et al. (2020). Dietary habits in adults during quarantine in the contet of COVID-19 pandemic. Obesity Medicine, v. 19, pp. 100254, 2020. https://doi.org//10.1016/j.obmed.2020.100254

Araújo, E. P. S., Ferreira, A. L. M., Fayh, A. P. T. \& Lima, S. C. V. C. (2020). Aspectos nutricionais na prevenção e tratamento de indivíduos com sintomas leves da COVID-19. https://repositorio.ufrn.br/jspui/bitstream/123456789/29246/5/_Terapia\%20nutricional\%20para\%20preven\%C3\%A7\%C3\%A3o\%2C\%20 tratamento\%20e\%20reabilita\%C3\%A7\%C3\%A3o\%20de\%20indiv\%C3\%ADduos\%20com\%20COVID-19.pdf/.

Araújo, L., Antonino, P., Chaves, C. \& Salgado, S. (2020). Como a alimentação pode melhorar a imunidade? Dicas para uma alimentação saudável durante a quarentena. Abril 2020. Universidade Federal de Pernambuco; Centro de Ciências da Saúde; Departamento de Nutrição; Serviço-Escola de Nutrição Emília Aureliano.

Barazzoni, R., Bischoff, S. C., Krznaric, Z., Pirlich, M. \& Singer, P. (2020). ESPEN expert statements and practical guidance for nutritional management of individuals with SARS-CoV-2 infection. Clin. Nutr (Internet). 2020 Mar 31; 1-8. doi: 10.1016/j.clnu.2020.03.022.

Bicalho, D. (2020). O Programa Nacional de Alimentação Escolar como garantia do direito à alimentação no período da pandemia do COVID-19. https://orcid.org/0000-0003-4484-6975

Brasiel, P. G. A. (2020). The key role of zine in elderly immunity: A possible approach in the COVID-19 crisis. Clinical Nutrition ESPEN, 2020. https://doi.org//10.1017/S1368980019002623

Brasil. Ministério da Saúde. (2014). Guia Alimentar para a população brasileira: promovendo a alimentação saudável. Brasília: Ministério da Saúde, 2014.

Brasil. Ministério da Saúde (2020). Painel Coronavírus - COVID-19. https://www.covid.saude.gov.ber.

Bhutani, S. \& Cooper, J. A. (2020). COVID-19 related home confinement in adults: weight gain risks and opportunities. Obesity (Silver Spring) [Internet]. 2020 May. https://doi.org/10.1002/oby.22904

Butler, M. et al. (2020). The impact of nutrition on COVID-19 susceptibility and long-term consequences. Brain, Bchavio, and Immunity, pp. 53-54, 2020.

Cabrera, M. P. C, et al. (2020). La colisión de das pandemias: COVID-19 y obesidad.

Cai, Q., Che, F., Wang, T., Luo, F., Liu, X., Wu, Q. \& Chen, J. (2020). Obesity and Covid-19 severity in a designated hospital in Shenzhen, China. Diabetes Care. Doi: 10.2337/dc20-0576.

Calder, P. C., Carr, A. C., Gombart, A. F. \& Eggersdorfer, M. (2020). Optimal Nutritional Status for a Well-Functioning Immune System Is an Important Factor to Protect against Viral Infections. Nutrients [Internet]. abril de 2020.12(4):1181. https://www.mdpi.com/2072-6643/12/4/1181

Claro, R. et al. (2015). Consumo de Alimentos não Saudáveis Relacionados a Doenças Crônicas não Transmissíveis no Brasil: Pesquisa Nacional de Saúde, 2013. Epidemiol. Serv. Saúde. v. 24, n. 2, 2015.

Conte, F. A. \& Schwengber, M. S. V. (2020). Saberes nutricionais econômicos midiáticos e o cuidado com a alimentação: light, diet e zero. Revista Espaço Acadêmico - n. 222 - maio./jun. 2020-bimestral.ano XX-ISSN n 1519.6186.

Costa, T. R. M., Correia, R. S., Silva, P. H. S., Barbosa, G. S. L., Oliveira, L. M. et al. (2020). A Obesidade como coeficiente no agravamento de pacientes acometidos por COVID-19. Reserd, Society and Development, v. 9, n. 9, e395997304, 2020 (CC BY 4.0)/ ISSN 2525-3409/ doi: dx.doi.org/10.33448/rsd-v9i9.

Demoliner, F. \& Daltoé, L. (2020). COVID-19: Nutrição e comportamento alimentar no contexto da pandemia. Perspectiva: Ciência e Saúde, Osório, V. 5 (2): 36-50, Ago 2020

Dias, M. J. L., Chini, M. C., Cardoso, T. F., Orrico, S. R. P. \& Pereira, B. L. B. (2020). COVID-19 e Nutrição. Ulakes Journal of Medicine. 1 (EE) 106-117 Durães, S. A., Souza, T. S., Gomes, Y. A. R. \& Pinho, L. (2020). Implicações da Pandemia da COVID-19 nos hábitos Alimentares. Unimontes Científica, Montes Claros (MG), Brasil, v. 22, n. 2 p. 1-20, jul/dez.2020. Eissn 2236-5257. 10.46551/ruc.v22n2a09

Elliston, K. G., Ferguson, S. G., Schuz, N. \& Schuz, B. (2017). Situational cues and momentary food environment predict everyday eating behavior in adults with overweight and obesity. Health Psychol [Internet]. 2017 Apr; 36(4):337-45. https://doi.org/10.1037/hea0000439

Elizabeth, L. et al. (2020). Ultra-Processed Foods and Health Outcomes: A Narrative Review. Nutrientes. v. 12, n. 7, junho, 2020. 
Li, S. et al. (2020). The Impact of COVID-19 Epidemic Declaration on Psychological Consequences: A Study on Active Weibo Users. Int. J. Environ. Res. Public Health. v. 17, n. 6, março, 2020.

Lima, S. C. V. C. (Organizadora) (2020). Terapia Nutricional para prevenção, tratamento e reabilitação de indivíduos com COVID-19. (Recurso eletrônico) Natal, RN: EDUFRN, 2020

Lopes, W. C. et al. (2020). Consumption Of Ultra-Processed Foods By Children Under 24 Months Of Age And Associated Factors. Rev. Paul. Pediatr., São Paulo, v. 38, e2018277, 2020. http://www.scielo.br/scielo.php?script=sci_arttext\&pid=S0103-05822020000100424\&lng=en\&nrm=iso. https://doi.org/ $10.1590 / 1984-0462 / 2020 / 38 / 2018277$

Louzada, M. L., Martins, A. P.; Canella, D. S., Baraldi, L. G., Levy, R. B., Claro, R. M. et al. (2015). Ultra-processed foods and the nutritional dietary profile in Brazil. Rev Saude Publica. 2015; 49:1-11.http://dx.doi.org/10.1590/S0034-8910.2015049006132

Malta, D. C., Szwarcwald, C. L., Barros, M. B. A., Gomes, C. S., Machado, I. E. et al. (2020). A pandemia da COVID-19 e as mudanças no estilo de vida dos brasileiros adultos: um estudo transversal, 2020. Epidemiol. Ser. Saúde, Brasília, 29(4):e2020407, 2020. Doi: 10.1590/s1679-49742020000400026

Martinez Steele, E., Rauber, F., Costa, C. S., Leite M. A., Gabe K. T., Louzada M. L., Levy, R. B. \& Monteiro C. A. (2020). Mudanças alimentares na coorte NutriNet Brasil durante a pandemia de covid-19. Rev Saude Publica. 2020;54:91.https://doi.org/10.11606/s1518-8787.2020054002950

Martinez-Ferran, M. et al.(2020). Metabolic Impacts of Confinement during the COVID-19 Pandemic Due to Modified Diet and Physical Activity Habits. Nutrients, v. 12, n. 6, p. 1549, 2020.

Martinotto, P. et al. (2020). Hábitos alimentares de trabalhadores de um Centro Universitário: mudanças durante a pandemia de COVID-19. VIII Congresso de Pesquisa e Extensão da FSG. http://ojs.fsg.br/index.php/pesquisaextensao.ISSN 2318-8014.

Mendes, L., Cebola, M., Mendes, D., Marinho, A. \& Guerreiro, A. S. (2020). Intervenção nutricional no doente com COVID-19. Saúde \& Tecnologia . MAIO /2020 / \#23/ P. 11-18. ISSN: 1646-9704.

Misumi, I., Starmer, J., Uchimura, T., Beck, M. A., Magnuson, T. \& Whitmire, J. K. (2019). Obesity expands a distinct population of T cells in adipose tissue and increases vulnerability to infection. Cell Rep. 2019 Apr 9;27(2):514-24

Monteiro, C. A., Levy, R. B., Claro, R. M., Castro, I. R. \& Cannon, G. (2010). Increasing consumption of ultra-processed foods and likely impact on human health: evidence from Brazil. Public Health Nutr.2010;14:5-13. https://doi.org/10.1017/S1368980010003241

Monteiro, C. A., Cannon, G., Lawrence, M., Costa Louzada, M. L. \& Pereira, M. (2019). Ultra-processed foods, diet quality, andhealth using the NOVA classification system [Internet].Rome: Food and Agriculture Organization of the United Nations; 2019. 44 p. http://www.fao.org/3/ca5644en/ca5644en.pdf

Montemurro, N. (2020). The emotional impact of COVID-19: from medical staff to common people. Brain Behav Immun. 2020.

Naja, F. \& Hamadeh, R. (2020). Nutrition amid the COVID-19 pandemic: a multi-level framework for action. European Journal of Clinical Nutrition, v. $74, \mathrm{n}$. 8, p. $1117-1121,2020$.

Oliveira, L. V., Rolim, A. C. P., Silva, G. F., Santos, N. R., Araújo, L. C., Braga, V. A. L. \& Coura, A. G. L. (2021). Modificações dos Hábitos Alimentares Relacionadas à Pandemia do Covid-19: uma Revisão de Literatura. Brazilian Journal of Health Review 2021, 4 (2): 8464-8477. doi: 10.34119/bjhrv4n2-367

Peçanha, T., Goessler, K. F., Roschel, H. \& Gualano, B. (2020). Social isolation during the COVID-19 pandemic can increase physical inactivity and the global burden of cardiovascular disease. Am J Physiol Heart Circ Physiol [Internet].2020Jun;318(6):1441-6. https://doi.org/10.1152/ajpheart.00268.2020.

Pellegrini, M., Ponzo, V., Rosato, R., Scumaci, E., Goitre, I., Benso, A., Bescatro, S., Crespi, C., Michieli, F.D., Ghigo, E., Broglio, F. \& Bo, S. (2020) Changes in Weight and Nutritional Habits in Adults with Obesity during the "Lockdown" Period Caused by the COVID-19 Virus Emergency. Nutrients, v. 12, n. 7, p. 2016, 2020.

Pereira, A. S. et al. (2018). Metodologia da Pesquisa Científica. [e-book]. 1 ed. Santa Maria/RS: UFSM, NTE.

Ribeiro, K. D. S., Garcia, L. R. S., Dametto, J. F. S., Assunção, D. G. F., Maciel, B. L. L. (2020). COVID-19 and nutrition: the need for initiatives to promote healthy eating and prevent obesity in childhood. Childhood Obes. 2020 Jun;16(4):235-7.

Ribeiro-Silva, R. C. et al. (2020). Implicações da pandemia COVID-19 para a segurança alimentar e nutricional no Brasil. Ciência e Saúde Coletiva, v. 25, n. 9, p. 3421-3430, 2020.

Rolland, B., Haesebaert, F., Zante, E., Amina, A. B., Haesebaert, J. \& Franck, N. (2020). Global changes and factors of increase in caloric food, screen and substance use during the early COVID-19 containment phase in France: a general population online survey. J. Med. Internet Res, 2020.

Rundle, A. G., Park, Y., Herbstman, J. B., Kinsey, E. W., Wang, Y. C. (2020). COVID-19-related school closings and risk of weight gain among children. Obesity (Silver Spring). 2020 Jun;28(6):1008-9.

Sallis, J. F., Adlakha, D., Oyeyemi, A. \& Salvo, D. (2020). An international physical activity and public health research agenda to inform COVID-19 policies and practices. J Sport Health Sci [Internet]. 2020 May :9(4):328-34. https://dx.doi.org/10.1016\%2Fj.jshs.2020.05.005

Schmidt, B., Crepaldi, M. A., Bolze, S. D. A., Neiva-silva, L. \& Demenech, L. M. (2020). Saúde Mental e Intervenções Psicológicas diante da pandemia do novo coronavirus (COVID-19). Estudos de Psicologia (Campinas), 37, e200063. http://dx.doi.org/10.1590/1982-0275202037e200063

Schweren, L. J. S., Larsson, H., Vinke, P. C., Li, L., Kvalvik, L. G., Arias-valquez, A., Haavik, J. \& Hartman, C. A. Diet quality, stress and common mental health problems: A cohort study of 121,008 adults. Clinical Nutrition, 2020. 
Research, Society and Development, v. 10, n. 7, e11610716602, 2021

(CC BY 4.0) | ISSN 2525-3409 | DOI: http://dx.doi.org/10.33448/rsd-v10i7.16602

Shekerdemian, L. S., Mahmood, N. R., Wolfe, K. K., Riggs, B. J., Ross, C. E., Mckiernan, C. A. et al. (2020). Characteristics and Outcomes of Children With Coronavirus Disease 2019 (COVID-19) Infection Admitted to US and Canadian Pediatric Intensive Care Units. JAMA Pediatr [Internet]. 2020 Mai 11. https://jamanetwork.com/journals/jamapediatrics/fullarticle/2766037

Sinha, I. P., Harwood, R., Semple, M. G., Hawcutt, D. B., Thursfield, R.; Narayan O. et al. (2020). COVID-19 infection in children. Lancet Respir Med [Internet]. 2020 Mai.8(5):446-7.https://linkinghub. elsevier.com/retrieve/pii/S2213260020301521

Sousa, G. C., Lopes, C. S. D., Miranda, M. C., Silva, V. A. A. \& Guimarães, P. R. (2020). A Pandemia de COVID-19 e suas repercussões na epidemia da obesidade de crianças e adolescentes. Revista Eletrônica Acervo Saúde/Eletronic Journal Collection Health/ ISSN 2178-2091. REAS/EJCH/ Vol. 12(12)/E4743/ DOI: https://doi.org/10.25248/reas.e4743.2020

Sociedade Brasileira de Pediatria. (2020). Obesidade em Crianças e Adolescentes e COVID-19. Nota de Alerta. Sociedade Brasileira de Pediatria. Grupo de Trabalho em Atividade Física. São Paulo: SBP, 2020.

Vasconcelos, F. A. \& Batista Filho M. (2011). History of the scientific field of Food and Nutrition in Public Health in Brazil. Cienc Saude Coletiva. 2011;16:81-90. http://dx.doi.org/10.1590/S1413-81232011000100012

Wang, C. et al. (2020). Immediate psychological responses and associated factors during the initial stage of the 2019 coronavirus disease (COVID-19) epidemic among the general population in China. Int J Environ Res Public Health. v. 17, n. 1729, 2020.

Zabetakis, I., Lordan, R., Norton, C. \& Tsoupras, A. (2020). COVID-19: The Inflammation Link and the Role of Nutrition in Potential Mitigation. Nutrients [Internet]. maio de 2020;12(5):1466.https://www.mdpi.com/2072-6643/12/5/1466

Zandifar, A. \& Badrfam, R. (2020). Iranian mental health during the COVID-19 epidemic. Asian Journal of Psychiatry, 51, 101990. http://dx.doi.org/10.1016/j.ajp.2020.101990.

Zang, L. et al. (2020). Potential interventions for novel coronavirus in China: A systematie review. Journal of Medical Virology, pp. 479-490, 2020. 